\title{
18 Interkulturelle Aspekte in der Notfallversorgung
}

\author{
Diana Kietzmann und Silke Schmidt
}

\subsection{Einführung}

Die Zahl der Migranten ist weltweit von 175 Millionen im Jahr 2000 auf 232 Millionen im Jahr 2013 gestiegen. Deutschland wurde nach den USA und der Russischen Föderation im Jahr 2013 als Land mit der dritthöchsten Aufnahme von Migranten geführt (United Nations General Assembly Trends in International Migrant Stock 2013). Der derzeitige Anteil der Bevölkerung mit Migrationshintergrund in Deutschland beträgt etwa 20\%, wobei die zahlenmäßig größten Gruppen Migranten aus der Türkei (18,3\%), gefolgt von Polen $(9,4 \%)$ und der Russischen Föderation $(7,4 \%)$ darstellen (Statistisches Bundesamt 2013). Die mit den Migranten einhergehende sprachliche und kulturelle Vielfalt steht für eine wertvolle gesamtgesellschaftliche Bereicherung. Gleichzeitig sind mit genannter Vielfalt Herausforderungen verbunden, die sich unter anderem im Bereich der Notfallversorgung zeigen. Studien verweisen auf vereinzelte Aussagen von Personal in Notfallaufnahmen, denen zufolge der Umgang mit Sprachbarrieren als schwierig empfunden wird, ebenso wie der Umgang mit einer Vielzahl von Angehörigen bei Notfallbetroffenen mit Migrationshintergrund (Hultsjo u. Hjelm 2005; Ozolins u. Hjelm 2003). Darüber hinaus deuten weitere vereinzelte Aussagen von Rettungsdienstpersonal darauf hin, dass kulturbedingte Unterschiede im Ausdruck von Schmerzempfindungen zu einer erschwerten Diagnose führen können (Machado 2010; Hoffmann 2012).

Professionelle Reaktionen auf die als schwierig empfundenen Situationen setzen sowohl Grundkenntnisse, als auch kulturspezifisches Wissen voraus. Letzteres darf aber nicht zu der Annahme führen, eine Auflistung konkreter Hinweise für den Um- 
gang mit Notfallbetroffenen in Abhängigkeit von ihren jeweiligen kulturellen Hintergründen zu erhalten. Zum einen können sich Normen und Werte sowie entsprechende Überzeugungen und Verhaltensmuster im Laufe der Zeit verändern. Zum anderen besteht die Gefahr von Stereotypen, denn Notfallbetroffene würden nicht in ihrer Individualität wahrgenommen werden, sondern als „türkischer Notfallpatient“ oder „russischer Notfallpatient“.

Um folgend das Thema „Interkulturelle Aspekte in der Notfallversorgung“ näher zu beleuchten, werden im Hinblick auf Grundkenntnisse der Begriff der Kultur näher erläutert, einhergehend mit dem Einfluss von Kultur auf das Verständnis von Gesundheit und Krankheit. Im Zusammenhang mit kulturspezifischen Themen werden anschließend die beschriebenen, als schwierig empfundenen Situationen thematisiert.

Eine Auflistung konkreter Hinweise für den Umgang mit Notfallbetroffenen in Abhängigkeit von ihren kulturspezifischen Hintergründen wäre nicht nur unrealistisch, sondern würde auch zu einer Förderung von Stereotypen beitragen.

\subsection{Die interkulturelle Notfallsituation}

Aufgrund der in Kapitel 18.1 beschriebenen Bevölkerungsstruktur Deutschlands können sich Notfallbetroffene in sprachlicher und kultureller Hinsicht vom Personal unterscheiden. Damit einhergehende Herausforderungen für das Personal sind ebenfalls in Kapitel 18.1 beschrieben und basieren unter anderem auf kulturbedingte Unterschiede im Verständnis von Gesundheit und Krankheit.

Diese sollten bereits im Vorfeld einer interkulturellen Notfallsituation erörtert werden mit dem Ziel auf genannte Herausforderungen adäquat reagieren zu können.

\subsection{Kultur}

\subsubsection{Der Begriff Kultur in der medizinischen Aus-, Fort- und Weiterbildung}

In der medizinischen Aus-, Fort- und Weiterbildung existiert eine Vielzahl von Definitionen des Begriffes Kultur. So beschreibt Leininger (1988) im Sinne klassischer anthropologischer Definitionen Kultur als „die erlernten, gemeinsamen übermittelten Werte, Überzeugungen, Normen und Lebensweisen einer bestimmten Gruppe, welche deren Überlegungen, Entscheidungen und Handlungen auf eine strukturierte Weise leiten“. Trotzdem dieser Inhalt nicht grundsätzlich in Frage zu stellen ist, entgegnen Kritiker, dass die genannte Definition die Unveränderlichkeit der genannten „Werte, Überzeugungen, Normen und Lebensweisen einer bestimmten Gruppe“ suggeriert (Dornheim 1997) und damit einhergehend die Annahme eines vermeintlich allgemein gültigen Bildes über den „türkischen Patienten“ beziehungsweise den „,russischen Patienten“. Andere Autoren (Guarnaccia u. Rodriguez 1996; Boutin-Foster et al. 2008) betonen daher, dass Kultur ebenso als dynamisches Phänomen betrachtet werden muss. Dahinter steht die Annahme, dass sämtliche Faktoren, die einen Einfluss auf Kultur haben, veränderbar sind und somit auch Werte, Überzeugungen, Normen und Lebensweisen einer bestimmten Gruppe. Diese Faktoren werden als so- 
ziokulturelle Faktoren bezeichnet und schließen unter anderem Bildungsstatus und Einkommen, aber auch Migrationserfahrungen mit ein (Betancourt et al. 2002).

Kultur ist ein dynamisches Phänomen. Werte, Überzeugungen, Normen und Lebensweisen können sich in Abhängigkeit von bestimmten Faktoren/Erfahrungen ändern.

\subsubsection{Der Begriff Kultur im Kontext der Notfallversorgung}

Kietzmann et al. (2014) haben im Zusammenhang mit der Erarbeitung konzeptioneller Grundlagen für die Vermittlung interkultureller Kompetenzen im Bereich der Notfallversorgung den Begriff der Kultur thematisiert und diesem folgende Inhalte zugrunde gelegt:

- Kulturelle und soziale Faktoren beeinflussen das Wahrnehmen, Denken und Handeln.

- Kultur ist komplex und steht im Zusammenhang mit der Summe der Erfahrungen eines Notfallbetroffenen. Eine Reduzierung von Kultur auf das Kriterium Herkunft ist ungenügend.

- Kultur als Ressource im Umgang mit Herausforderungen: Die vom Rettungspersonal unter Umständen als problematisch wahrgenommenen Verhaltensweisen können jedoch für Notfallbetroffene mit Migrationshintergrund im Umgang mit einer Herausforderung (überlebens-)wichtig sein.

Betont wird die Summe der individuellen Erfahrungen, die das Wahrnehmen, Denken und Handeln (in Notfallsituationen) prägen. Gleichzeitig wird darauf verwiesen, dass ein Erfragen beziehungsweise die Betrachtung sämtlicher individueller Erfahrungen eines Notfallbetroffenen nicht möglich ist.

Überlegungen, Entscheidungen und Handlungen von Notfallbetroffenen (mit Migrationshintergrund) können über kulturelle Hintergründe hinaus auf jeweils individuell unterschiedlichen Erfahrungen basieren.

\subsubsection{Der Einfluss von Kultur auf das Verständnis von Gesundheit und Krankheit}

Kulturell bedingt unterschiedliche Auffassungen von Gesundheit und Krankheit können sich für das medizinische Personal in unerwartete oder gar irritierende Verhaltensweisen von Notfallbetroffenen mit Migrationshintergrund äußern. Das Anliegen, solche Verhaltensweisen besser verstehen zu können, ist häufig mit konkreten Fragen verbunden: „Wie äußern türkische Notfallbetroffene starke Schmerzen in der Nierengegend?“ oder „Wie ist die Einstellung russischer Notfallbetroffener gegenüber diesen oder jenen Untersuchungen?" Wie bereits angedeutet, kann es keine allgemeingültigen Antworten darauf geben. Innerhalb der Bevölkerungsgruppen türkischer und russischer Abstammung können der Ausdruck von Schmerzempfindungen 
oder die Einstellung gegenüber verschiedener Untersuchungen in Abhängigkeit von der Summe der individuellen Erfahrungen sehr heterogen sein. Dennoch ist es wichtig, über Grundkenntnisse im Zusammenhang mit unterschiedlichen Auffassungen von Gesundheit und Krankheit zu verfügen, da der Zusammenhang zwischen Kultur, Gesundheit und Krankheit vielfach beschrieben wurde (Helman 2007).

\section{Der Einfluss von Kultur auf das Verständnis von Gesundheit und Krankheit ist vielfach beschrieben. Ungeachtet dessen kann dieses Verständnis zwischen An- gehörigen einer Kultur variieren.}

Da die zahlenmäßig größte Gruppe der Migranten aus der Türkei (18,3\%) stammt (Statistisches Bundesamt 2013), wird folgend der Zusammenhang zwischen einer islamisch geprägten Kultur, Gesundheit und Krankheit exemplarisch dargestellt.

Gesundheit zählt im islamischen Verständnis zu den wichtigsten Gottesgaben und wird als hohes Gut verstanden (Ilkilic 2007). Die mit dem Gottesgabenverständnis einhergehende Verantwortung impliziert sowohl Maßnahmen im Zusammenhang mit einer gesunden Lebensführung, als auch bestimmte Interventionen bei Vorhandensein einer Krankheit (Ilkilic 2005).

Im Zusammenhang mit Krankheit ist zunächst festzuhalten, dass diese in islamisch geprägten Kulturen als ganzheitlich angesehen wird. Das bedeutet, dass nicht ein einzelnes Organ oder Körperteil von einer Krankheit betroffen ist und behandelt werden muss, sondern „der ganze Mensch“ (von Bose u. Terpstra 2012). So können für lokale, örtlich begrenzte Beschwerden mitunter Beschreibungen wie „alles kaputt“ oder „ganzer Körper krank“ benutzt werden (Kielhorn 1996). Des Weiteren ist ein kollektives Verständnis von Krankheit vorhanden. Das heißt, dass die Krankheit nicht nur den Erkrankten betrifft, sondern die gesamte Familie (von Bose u. Terpstra 2012). Die daraus resultierende hohe Anteilnahme von Angehörigen ist gleichsam mit der Annahme verbunden, dass menschliche Nähe und Wärme zur Genesung des Erkrankten beitragen (von Bose u. Terpstra 2012).

In Hinblick auf Krankheitswahrnehmung und -bewältigung geben Studien ebenfalls Hinweise auf kulturbedingte Unterschiede (Franz et al. 2007; Berg 2001). Unter anderem hat Kizilhan (2009) folgende Syndrome auf Grundlage einer Befragung türkischstämmiger Patientinnen und Patienten aufgeführt, deren Bezeichnung, Ätiologie und Beschwerdebild in Tabelle 23 verkürzt dargestellt werden.

Die Zuordnung von verschiedenen Beschwerden zu spezifischen Organen deutet auf Unterschiede im Anatomieverständnis hin. Dies wird durch die Feststellung gestützt, dass die Betroffenen nicht-körperliche Beschwerden so beschreiben als seien diese somatisch (Kizilhan 2009). Beispielsweise und wie in Tabelle 23 ersichtlich, ist eine brennende Leber häufig der Ausdruck für Traurigkeit, Sorgen und schweres Leid.

Einhergehend mit diesem Anatomieverständnis unterscheiden sich Schmerzempfindungen und-ausdruck ebenfalls. Kohnen (2003) beschreibt mit Blick auf familienorientierte Gesellschaften ein emotionaleres Schmerzerleben. Das heißt, dass die mit dem Schmerzerleben einhergehenden emotionalen Faktoren gegenüber den somatischen Faktoren stärker betont werden. Hinzu kommt die bereits vorab beschriebene kollektive/familiäre Bewältigung vor dem Hintergrund der Überzeugung, dass menschliche 
Tab. 23 Kulturspezifische Syndrome nach Kizilhan (2009), (erweitert nach Petersen [1995])

\begin{tabular}{|c|c|c|}
\hline Bezeichnung & Ätiologie & Beschwerdebild \\
\hline Nabelfall & $\begin{array}{l}\text { schwer heben, in die Höhe springen, } \\
\text { schwere Arbeit, schweres Leben, Stress }\end{array}$ & $\begin{array}{l}\text { Bauch-/Magenschmerzen, Übelkeit, } \\
\text { Schwindel, Schwäche, Müdigkeit }\end{array}$ \\
\hline Brennende Leber & Traurigkeit, Sorgen, schweres Leid & Leberschmerzen, Oberbauchschmerzen \\
\hline Rückenschmerzen & $\begin{array}{l}\text { Familiäre Konflikte, Sorgen, Rollen- } \\
\text { problematik, schwere Arbeit }\end{array}$ & $\begin{array}{l}\text { stechende und ziehende Rückenschmer- } \\
\text { zen, kaum in der Lage, sich zu bewegen } \\
\text { oder etwas zu heben und zu tragen }\end{array}$ \\
\hline $\begin{array}{l}\text { Wanderschmerzen/ } \\
\text { Windschmerzen }\end{array}$ & Kummer, Sorgen, Konflikte, Sehnsucht & $\begin{array}{l}\text { Müdigkeit, Schwäche, Antriebslosigkeit, } \\
\text { jeden Tag befinden sich die Schmerzen } \\
\text { an einer anderen Stelle des Körpers }\end{array}$ \\
\hline Beklemmungsgefühl & $\begin{array}{l}\text { Kummer, Sorgen, Schuldgefühle, } \\
\text { Sehnsucht, Ärger }\end{array}$ & $\begin{array}{l}\text { Kopf- und Halsschmerzen, Enge-, } \\
\text { Globus- und Erstickungsgefühl, } \\
\text { Kurzatmigkeit }\end{array}$ \\
\hline
\end{tabular}

Nähe und Wärme zur Genesung des Erkrankten beitragen (von Bose u. Terpstra 2012). Dies setzt voraus, dass die Erkrankung beziehungsweise das damit einhergehende Schmerzerleben gegenüber den Angehörigen deutlich artikuliert werden sollte (Kohnen 2003).

Ein weiterer Unterschied bezieht sich auf das Schamgefühl, dem in der islamischen Religion ein Verständnis von körperlicher Unversehrtheit und Intimität zugrunde liegt(Ilkilic 2007). Gemäß diesem Verständnis kann ein Körperkontakt zwischen nicht verheirateten oder nicht verwandten Frauen und Männern als Intimitätsverletzung wahrgenommen werden (Ilkilic 2007).

Mögliche kulturbedingte Unterschiede, die im Rahmen einer Diagnose oder medizinischen Behandlung in Betracht gezogen werden müssen, betreffen unter anderem das Anatomieverständnis, das Schmerzempfinden und den Schmerzausdruck sowie das Schamgefühl. Darüber hinaus werden unterschiedliche Strategien in Hinblick auf die Bewältigung von Erkrankungen genutzt.

\subsection{Mögliche Herausforderungen in interkulturellen Notfallsituationen}

Mit den in Kapitel 18.3.3 beschriebenen kulturbedingten Unterschieden im Verständnis von Gesundheit und Krankheit wurden bereits Anhaltspunkte geliefert, die ein entsprechendes Verhalten von Notfallbetroffenen erklären. Doch wie kann im Bereich der Notfallversorgung mit den als schwierig empfundenen Situationen umgegangen werden? Und wie kann das unter Berücksichtigung des akuten Handlungsdrucks für das Personal geschehen? Wichtig ist zunächst, auf Basis von Grundkenntnissen ein Verständnis für kulturbedingt unerwartete oder gar irritierend erscheinende Verhaltensweisen zu entwickeln. Ebenso von Bedeutung ist, auf Basis der Grundkenntnis- 
se Hinweise für die Praxis zu erörtern. Vor diesem Hintergrund werden im Folgenden die eingangs als schwierig empfundenen Situationen thematisiert.

\subsubsection{Umgang mit einer Vielzahl von Angehörigen}

Wie bereits beschrieben, ist die Begleitung eines Notfallbetroffenen durch eine Vielzahl von Angehörigen mit der Annahme einer familiären/kollektiven Krankheitsbewältigung verbunden (von Bose u. Terpstra 2012). Aufgrund der Bedeutsamkeit dieser Annahme sollte den Rahmenbedingungen entsprechend vereinzelten Angehörigen eine Begleitung des Notfallbetroffenen ermöglicht werden.

Um eine Vielzahl von Angehörigen auf vereinzelte Angehörige zu begrenzen, kann zum einen die/der Notfallbetroffene gefragt werden, wessen Anwesenheit gewünscht ist. Ist dies aufgrund der Schwere des Notfalls nicht möglich, kann bei den Angehörigen selbst erfragt werden, wer den Notfallbetroffenen begleiten möchte und die Funktion des Ansprechpartners (für das Personal/für die anderen Angehörigen) übernehmen möchte.

\subsubsection{Unterschiede im Schmerzempfinden und -ausdruck}

Um auf Herausforderungen im Zusammenhang mit Unterschieden im Schmerzempfinden/-ausdruck besser eingestellt/vorbereitet zu sein, sind Grundkenntnisse nötig. Diese beziehen sich unter anderem auf die in Kapitel 18.3.3 erwähnten Unterschiede im Anatomieverständnis.

Darüber hinaus sollte das bereits beschriebene ganzheitliche Verständnis von Krankheiten/Behandlungen bedacht werden, einschließlich die Benutzung von Schmerzausdrücken, die sich unabhängig vom Vorhandensein lokaler, örtlich begrenzter Beschwerden auf den ganzen Körper beziehen können (Kielhorn 1996).

Über die genannten Bezeichnungen und das beschriebene Verständnis hinaus sind eine Vielzahl weiterer Unterschiede im Schmerzempfinden und -ausdruck vorhanden. Folglich wäre ein gänzliches Wissen darüber unrealistisch, und zu bedenken, dass das Verhalten von Notfallbetroffenen höchst unterschiedlich sein kann in Abhängigkeit von der Summe der jeweils individuellen Erfahrungen. Daher ist von Bedeutung, Etikettierungen wie „Mamma Mia Syndrom“ oder „Morbus Bosporus“ zu hinterfragen. Die Verwendung dieser steht im Zusammenhang mit Unkenntnis über kulturell bedingt unterschiedliche Auffassungen von Gesundheit und Krankheit (Bolten 2007).

\subsubsection{Akzeptanz von Personal des anderen Geschlechts}

Über die beschriebenen, als schwierig empfundenen Situationen hinaus wird die Akzeptanz von medizinischem Personal des anderen Geschlechts wiederholt thematisiert (Ilkilic 2007; von Bose u. Terpstra 2012). Dabei wird, unter der Voraussetzung der Realisierbarkeit, die Behandlung muslimischer Patientinnen und Patienten durch Personal desselben Geschlechts empfohlen (Ilkilic 2007). Ist die Realisierbarkeit dessen nicht gegeben, gilt aufgrund des Ausnahmezustands, der durch einen Krankheitsfall gegeben sein kann, das Prinzip: „Die Notlage macht das Verbotene erlaubt“ (Ilkilic 2005). 
Ungeachtet dessen gilt es, einige Aspekte zu berücksichtigen. Insgesamt sollten notwendige Behandlungsmaßnahmen transparent dargestellt und wenn möglich begründet werden. Im Zusammenhang mit konkreten Behandlungsmaßnahmen, die ein Abnehmen des Kopftuches und/oder die Entkleidung einer muslimischen Notfallbetroffenen erfordern, gibt es folgende Empfehlungen: Bei der Abnahme des Kopftuches sollte der Notfallbetroffenen selbst oder einer Angehörigen die Möglichkeit eingeräumt werden, dies zu tun (May u. Mann 2005). Nach Beendigung der Behandlungsmaßnahme sollte das Kopftuch oder ein Äquivalent (zum Beispiel Dreieckstuch) wieder aufgesetzt werden dürfen (May u. Mann 2005). Die Entkleidung einer muslimischen Notfallbetroffenen sollte, wenn gewünscht im Beisein einer Angehörigen erfolgen. Der Hintergrund ist, dass die Notfallversorgung ohne Beistand durch Angehörige die Ehre der Notfallbetroffenen verletzten könnte (May u. Mann 2005).

\subsubsection{Sprachbarrieren}

In Hinblick auf den Umgang mit Sprachbarrieren wird wiederholt empfohlen, auf Sprachmittler zurückzugreifen, die entweder professionell tätig sind oder ad hoc agieren (Ilkilic 2007; Brach u. Fraser 200o). Letztere können sowohl das Personal als auch Fremde, die zufällig in der Nähe sind, einschließen. Des Weiteren kann die Rolle eines Sprachmittlers durch Familienangehörige ausgeübt werden. Allerdings ist hier zu berücksichtigen, dass weibliche Notfallbetroffene mit Migrationshintergrund wichtige Informationen aus Scham zurück halten können, wenn ein männliches Mitglied der Familie sprachlich vermittelt.

Ebenso denkbar sind Broschüren, die relevante (Notfall-)Begriffe und/oder Patienteninformationen in verschiedenen Sprachen enthalten. Derzeit sind jedoch keine Übersetzungen bekannt, die in Notfallsituationen entsprechend genutzt werden können.

\subsection{Fazit}

Vor dem Hintergrund der Bevölkerungsstruktur Deutschlands erfordert der komplexe Arbeitsbereich der Notfallversorgung neben fachlichen auch interkulturelle Kompetenzen. Letztere setzen, wie eingangs beschrieben, sowohl Crundkenntnisse, als auch kulturspezifisches Wissen voraus. Auf Grundlage dessen kann zum einen ein Verständnis für ein kulturell bedingt unterschiedliches Verständnis von Gesundheit und Krankheit gefördert werden. Zum anderen kann die Beachtung daraus resultierender Hinweise für die Praxis zu einer besseren Bewältigung der als schwierig empfundenen Situationen beitragen.

\section{Literatur}

Berg G (2001) Subjektive Krankheitskonzepte - eine kommunikative Voraussetzung für die Arzt-Patientin-Interaktion?. In: David M, Borde T, Kentenich H (Hrsg.) Migration und Gesundheit. Zustandsbeschreibung und Zukunftsmodelle. Mabuse-Verlag, Frankfurt am Main.

Betancourt IR, Green AR, Carrillo El (2002) Cultural Competence in Health Care: Emerging Frameworks and Practical Approaches. The Commonwealth Fund

Bolten I (2007) Internationalisierung und interkulturelle Kompetenz im Gesundheitssektor. Kosmetische Medizin 1: 16-22 
Boutin-Foster C, Foster IC, Konopasek L (2008) Viewpoint: Physician, Know Thyself: The Professional Culture of Medicine as a Framework for Teaching Cultural Competence. Academic Medicine 83(1): 106-111

Brach C, Fraser I (2000) Can cultural competency reduce racial and ethnic health disparities? A review and conceptual model. Med Care Res Rev 57 Suppl 1: 181-217

Dornheim I (1997) Unterschiedliche Kulturbegriffe und ihre Bedeutung für Theorien der transkulturellen Pflege Ein Beitrag zu den Grundlagen der Pflegewissenschaft: In: Uzarewicz C, Piechotta G (Hrsg.) Transkulturelle Pflege. Verlag für Wissenschaft und Bildung, Berlin, S. 11-32

Franz M, Lujić C, Koch E, Wüsten B, Yürük N, Gallhofer B (2007) Subjektive Krankheitskonzepte türkischer Migranten mit psychischen Störungen - Besonderheiten im Vergleich zu deutschen Patienten. Psychiatrische Praxis 34: 332-338

Guarnaccia P, Rodriguez 0 (1996) Concepts of culture and their role in the development of culturally competent mental health services. Hispanic Journal of Behavioral Sciences 18(4): 419-443

Helman CG (2007) Culture, Health and Illness. Fifth edition. Taylor \& Francis

Hoffmann N (2012) Interkulturalität in der Notfallrettung: Der Umgang mit muslimischen Patienten - Fallbeispiele der Berliner Feuerwehr 2012. Europa Universität Viadrina, Frankfurt/Oder

Hultsjo S, Hjelm K (2005) Immigrants in emergency care: Swedish health care staff's experiences. Int Nurs Rev 52(4): $276-85$

Ilkilic I (2005) Gesundheitsverständnis und Gesundheitsmündigkeit in der islamischen Tradition. Medizinethische Materialien, Bonn

Ilkilic I (2007) Medizinethische Aspekte im Umgang mit muslimischen Patienten [Medical ethical aspects of culture in social interactions with Muslim patients]. Dtsch Med Wochenschr 132: 1587-1590

Kielhorn R (1996) Krank in der Fremde. Psychosozial 63: 15-27

Kietzmann D, Hannig C, Kehl D, Schmidt S (2014) Der Kulturbegriff aus Sicht von Betroffenen mit Migrationshintergrund und Bevölkerungsschutzexperten im Kontext der Notfallversorgung. Psychother Psych Med 68(08): 309-314

Kizilhan II (2009) Subjektive Krankheitswahrnehmung bei MigrantInnen aus familienorientieren Gesellschaften. In: H.B. Stiftung (Hrsg.) Migration \& Gesundheit

Kohnen N (2003) Von der Schmerzlichkeit des Schmerzerlebens. Wie fremde Kulturen Schmerzen wahrnehmen, erleben und bewältigen. pvv, Ratingen

Leininger MM (1988) Leininger's theory of nursing: cultural care diversity and universality. Nurs Sci Q 1(4): 152-60

Machado C (2010) Interkulturelle Kommunikation in der präklinischen Notfallmedizin: Eine empirische Studie bei der Berufffeuerwehr Hamburg. VDM Publishing

May AT, Mann R (2005) Soziale Kompetenz im Notfall. Praxisanleitung nicht nur für den Rettungsdienst - ein Unterrichtskonzept. Münster, Lit Verlag

Ozolins L, Hjelm K (2003) Nurses' experiences of problematic situations with migrants in emergency care in Sweden. Clinical Effectiveness in Nursing 7(2): 84-93

Petersen A (1995) Somatisieren die Türken oder psychologisieren wir? Gedanken zur angeblichen Neigung der Türken zum Somatisieen. Curare 18(2): 531-540

Statistisches Bundesamt (2013) Bevölkerung und Erwerbstätigkeit. Bevölkerung mit Migrationshintergrund - Ergebnisse des Mikrozensus 2012

United Nations General Assembly Trends in International Migrant Stock (2013) The 2013 Revision

von Bose A, Terpstra I (2012) Muslimische Patienten pflegen. Praxisbuch für Betreuung und Kommunikation. Berlin, Heidelberg, Springer 


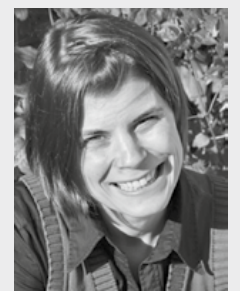

\section{Diana Kietzmann, Dipl.-Psych.}

Studium der Sozialen Arbeit und Psychologie. Seit 10/2010 Tätigkeit als wissenschaftliche Mitarbeiterin des Lehrstuhls Gesundheit und Prävention am Institut für Psychologie der Ernst-Moritz-Arndt-Universität Greifswald. Wissenschaftlicher Schwerpunkt unter anderem im Bereich der Vermittlung interkultureller Kompetenzen.

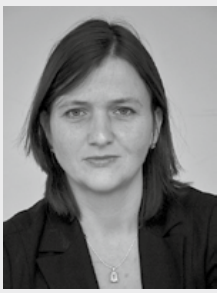

\section{Prof. Dr. phil. Silke Schmidt}

Studium der Psychologie, Promotion an der Friedrich-Schiller-Universität Jena 2001, Habilitation am Universitätsklinikum Hamburg 2006. Seit 1/2009 W3Professur und Leiterin des Lehrstuhls Gesundheit und Prävention am Institut für Psychologie der Ernst-Moritz-Arndt-Universität Greifswald, Leiterin vieler multizentrischer Studien zur psychischen Prävention im Bereich Gesundheit und Bevölkerungsschutz. 